\title{
REVISÃO SISTEMÁTICA DA LITERATURA SOBRE ATENDIMENTOS EM SAÚDE PÚBLICA SOB A PERSPECTIVA DE PESSOAS TRANSGÊNEROS
}

\section{CARLA RIBEIRO COHEN 1, RAFAEL DE TILIO 2}

1., 2. UNIVERSIDADE FEDERAL DO TRIÂNGULO MINEIRO, Uberaba/MG, Brasil

\begin{abstract}
Objective: The purpose of this study was to perform a systematic literature review of Brazilian and international scientific literature (published in Brazil) between 2008 and 2018 method: Using the PICO (acronym for Patient, Intervention, Comparison, Outcome) strategy for systematic reviews of the scientific literature questioning how transgender people perceive the care received at the public health service. The databases used were SciELO, Lilas, Medline, PePSIC and APA PsycNET by combining terms related to the topic. Results: A total of 5,336 sources were retrieved and this total was refined according to the authors inclusion and exclusion criteria which include transgender population in their research and answer the guiding question. Thus, four articles were fully retrieved for analysis (thematic content analysis) in three thematic categories: SUS (Brazilian public health care system) care, use of social name, sexual infections and AIDS. Conclusion: Results highlight the difficulties of this population regarding the consolidation of their rights in public health care. The importance of further studies on the perception of transgender people about care in public health services is emphasized.
\end{abstract}

KEY WORDS: Transgender persons, transsexuals, health, health system.

\section{CÓMO CITAR / HOW TO CITE}

Ribeiro Cohen, C., \& De Tilio R. (2019). Revisão sistemática da literatura sobre atendimentos em saúde pública sob a perspectiva de pessoas transgêneros. Salud \& Sociedad, 10(3), 240-254. doi: 10.22199/issn.0718-7475-2019-03015 
$\mathrm{Na}$ perspectiva binária e heterossexual, masculinidade(s) e feminilidade(s) são compreendidas como a expressão cultural da diferença natural (biológica) dos sexos e que, em decorrência disso, todos os elementos constitutivos ou de expressão dos sujeitos estão condicionados a essa determinação (Bento, 2017). Neste sentido, para Butler (2016), o gênero segundo a heterossexualidade compulsória seria uma forma de regulação social, uma performance com consequências claramente normativas, pois habitualmente são punidos os que não desempenham corretamente o seu gênero e rompem com as prédicas do binarismo.

A heteronormatividade associada ao binarismo de gênero produz sujeitos e gêneros coerentes, reiterando que "o gênero é uma forma de regulação social" (Arán \& Peixoto Júnior, 2007, p.132), haja vista que a atribuição de sexos aos corpos também deve ser compreendida como uma experiência condicionada pelas significações culturais (Bento, 2017). Para Bento (2017) não existe um corpo livre de investimentos discursivos e a primeira intervenção que construiu 0 corpo-sexuado vincula o destino desse corpo à genitália, dirigindo o corpo/sexo a um desempenho condizente com o gênero naquilo que se denomina experiência cisgênera. Assim, 0 gênero adquire relevância através das roupas que compõem esse corpo, dos gestos, dos olhares, ou seja, de uma norma definida como apropriada. São estes sinais exteriores que estabilizam e dão visibilidade ao corpo generificado (Bento, 2017).

Neste sentido, as transgeneridades (isto é, os que rompem com a adequação entre sexo e gênero e que compreendem, dentre outros, as pessoas transexuais e as travestis) são igualmente construções identitárias possíveis que representam respostas aos conflitos gerados por uma ordem dicotomizada, naturalizada e imposta para os gêneros. São múltiplas as violências reais e simbólicas cometidas contra os transgêneros, irradiando a convicção de que são inferiores e/ou doentes aprisionados na sua própria posição existencial, por isso, cruzar os limites da cisgeneridade é colocarse em uma posição de risco.

Considerar "gênero [como] o mecanismo pelo qual as noções de masculino e feminino são produzidas e naturalizadas" (Butler, 2014, p. 253) é afirmar que existem regras, leis, interdições e punições diante de uma norma estabelecida, no caso, a cisgeneridade. Portanto, as principais pautas das lutas dos transgêneros são o direito legal à autodeterminação de identidade de gênero, o respeito a sua condição de ser humano e a utilização do nome social (Bento, 2017), além do acesso (universal, integral e com equidade) ao Sistema Único de Saúde (SUS) tal como preconizado pelo Estado brasileiro (Brasil, 1988) com o intento de promover a saúde, prevenir agravos e recuperar doentes.

No que diz respeito às demandas dos transgêneros, a Carta dos Direitos dos Usuários do SUS (Brasil, 2006) assegura não só o preenchimento do nome social nos documentos de identificação do SUS (Cartão Nacional de Saúde/CNS) como também explicita o direito ao cuidado, ao tratamento e ao atendimento no âmbito do SUS, livres de discriminação por orientação sexual e identidade de gênero, vetando o uso de formas desrespeitosas e preconceituosas no atendimento (Brasil, 2006).

Segundo Lionço (2009) a recusa em considerar as especificidades em Saúde dos transgêneros cercea o reconhecimento das variações sociais, subjetivas e biológicas do ser humano - como, por exemplo, a transexualidade e a travestilidade que demandam cuidados específicos quando comparados aos cisgêneros.

Para Melo, Perilo, Braz e Pedrosa (2011) a necessidade de sensibilização por parte de profissionais da Saúde para 0 atendimento não discriminatório da população lésbica, gay, bissexual e transexual (LGBT) ainda é tema recorrente nos planos, programas e documentos que apresentam diretrizes, 
objetivos e metas para as políticas públicas de saúde formuladas pelo Governo Brasileiro. Isso ocorre em decorrência da dificuldade em assegurar 0 atendimento de qualidade e livre de preconceito e discriminação para as populações LGBT, mesmo diante das garantias constitucionais de que todos os cidadãos no campo da Saúde devem ter garantidas suas especificidades num sistema universal, integral e equitativo (Calderaro, Fernandes, \& Mello, 2008).

Deste modo faz-se necessário uma ampliação da oferta de saúde e cuidados aos transexuais para além do processo transexualizador, além da desmistificação de que as travestis e transexuais buscam os serviços de saúde somente para a prevenção e/ou combate a infecções sexualmente transmissíveis (IST), incluindo HIVIAIDS. A partir do exposto, o presente estudo tem como objetivo apresentar uma revisão da literatura científica nacional e internacional sobre como os transgêneros percebem 0 atendimento realizado no serviço público de saúde.

\section{MÉTODO}

A revisão sistemática de literatura é um método que tem como finalidade sintetizar resultados obtidos em pesquisas sobre um tema ou questão, de maneira sistemática, ordenada e abrangente (Ercole, Melo, \& Alcoforado, 2014). Ela envolve a definição de um problema, a busca e a avaliação crítica das evidências disponíveis, a implementação das evidências na prática e a avaliação dos resultados obtidos (Mendes, Silveira, \& Galvão, 2008). A revisão sistemática também determina o conhecimento sobre uma temática específica, pois permite identificar, analisar e sintetizar resultados de estudos independentes sobre um assunto (Souza, Silva, \& Carvalho, 2010).

Neste sentido a questão norteadora do estudo foi construída com base na estratégia PICO (acrônimo das palavras Paciente, Intervenção, Comparação e
Outcome/Desfecho) desenvolvida no final da década de 1940 por pesquisadores ingleses, estadunidenses e canadenses da área da saúde cuja intenção era melhor fundamentar a produção do conhecimento científico baseado evidências (isto é, calcados em conhecimentos resultantes de pesquisas empíricas), e que é amplamente utilizada em estudos de revisão sistemática (Ercole, Melo, \& Alcoforado, 2014) que, se bem construída, possibilita a definição correta de quais informações ou evidências são necessárias para a resolução da questão de pesquisa, maximizando a recuperação das evidências nas bases de dados, focando no escopo da pesquisa e com isso evitando a realização de buscas desnecessárias (Santos, Pimenta, \& Nobre, 2007).

Valendo-se desta premissa, a pergunta norteadora delineada na presente revisão, a partir da estratégia PICO, foi: Como pessoas transgênero $(P)$ percebem 0 atendimento $(O)$ no serviço público de saúde (I)? Considerando que o objetivo da pesquisa não envolveu a comparação entre cenários ou técnicas, a estratégia PICO foi implementada na presente revisão sem 0 critério 'C' de comparação.

Para delinear o estudo e a seleção dos artigos foram definidos critérios de inclusão e de exclusão. Os de inclusão foram: ser apenas artigos publicados entre janeiro de 2008 a abril de 2018; artigos em português, inglês e espanhol; publicados em periódicos indexados e disponibilizados na íntegra e gratuitamente; com temática pertinente aos objetivos da revisão; e que respondessem à questão norteadora do estudo. Os critérios de exclusão foram: fontes de publicação que não fossem artigos, como monografias, teses, dissertações, livros, capítulos de livro e editoriais; artigos de revisão de literatura; artigos não pertinentes à temática; e artigos que não correspondentes à questão norteadora. Assim, os resumos condizentes com estes critérios foram selecionados e recuperados para leitura na íntegra. 
Para o levantamento dos artigos na literatura foi realizada, em abril de 2018, uma busca nas seguintes bases de dados: Scientific Electronic Library Online (SciELO), Literatura Latino-Americana e do Caribe em Ciências da Saúde (LILACS) através da Biblioteca Virtual em Saúde (BVS), Medical Literature Analysis and Retrieval System online (Medline) através da Biblioteca Virtual em Saúde (BVS), Periódicos Eletrônicos em Psicologia (PePSIC) e American Psychological Association (APA PsycNET).

É importante ressaltar que os unitermos empregados na busca dos artigos foram selecionados previamente nos Descritores em Ciência da Saúde (DeCS) da BVS a partir da pergunta norteadora. Deste modo, para a revisão, foram utilizados os seguintes unitermos nas línguas portuguesa e inglesa e os operadores booleanos AND e OR da seguinte maneira:

1) transexualismo $O R$ transsexualism AND assistência à saúde $O R$ delivery of health care

2) pessoas transgênero $O R$ transgender persons AND assistência à saúde OR delivery of health care

2.1) pessoas transgênero $O R$ transgender persons AND saúde OR health;

3) identidade de gênero OR gender indentity AND sistema único de saúde $O R$ unified health system

4) pessoas transgênero $O R$ transgender persons AND sistema único de saúde OR unified health system

5) transexuais OR transsexuals AND sistema único de saúde $\mathrm{OR}$ unified health system;

6) pessoas transgênero $O R$ transgender persons AND direito OR law

6.1) pessoas transgênero $O R$ transgender persons AND direito OR law AND sistema único de saúde OR unified health system 6.2) pessoas transgênero $O R$ transgender persons AND direito à saúde $O R$ right to health $A N D$ sistema único de saúde OR unified health system;

7) transexuais OR transsexuals AND direito OR law AND sistema único de saúde $O R$ unified health system.

O processo de busca e seleção foi realizado por dois juízes independentes, ambos com formação em Psicologia e experiência nesta estratégia metodológica. Após a leitura na íntegra dos artigos recuperados ao final de todas as etapas (descritas na Figura 1), as seguintes informações foram alocadas em uma planilha de editor de texto para posterior análise (apresentadas nas Tabelas 1 a 3): identificação do periódico, lugar, objetivo da pesquisa, desenho da pesquisa, número de participantes, população transgênero do estudo, coleta de dados (local e forma), idade dos participantes, período da pesquisa, título dos artigos, autores, periódicos, ano de publicação, principais resultados e categorias agrupadas por semelhança temáticas.

\section{RESULTADOS E DISCUSSÃO}

As buscas iniciais conduziram aos seguintes resultados em termos de quantidade de arquivos encontrados: SciELO $(n=735)$, LILACS ( $n=48)$, MedLine ( $n=1331)$, PsycNET $(n=505)$ e PePSIC $(n=2717)$ totalizando 5336 fontes recuperadas que foram refinadas seguindo os critérios de inclusão e exclusão estabelecidos. Tomando como base os critérios de inclusão 5212 artigos foram excluídos pelo título ( $n=5081$ ), pela repetição na mesma base $(n=56)$, por não serem artigos $(n=69)$, ou pelo idioma $(n=6)$ - uma vez que as bases de dados Psyclnfo e Pepsic não possuem filtros específicos para os dois últimos casos. Assim, foram incluídos pelo título 124 artigos dos quais 18 artigos foram excluídos por não serem disponibilizados integralmente e um foi excluído por não ser possível sua recuperação de forma integral nas bases de dados. Deste modo, restaram 105 artigos para a leitura dos resumos e, com base nos critérios de exclusão, 79 foram excluídos (22 
foram descartados por não serem artigos e 57 foram descartados por não responder à pergunta norteadora da pesquisa).

Assim, pelos critérios de inclusão restaram 26 artigos para leitura na íntegra, dentre os quais quatro foram excluídos por que se repetirem em bases diferentes e 18 artigos foram excluídos por não responderem à questão norteadora da pesquisa (dois estudos abordavam questões de violência mas não possuíam como cenário 0 sistema público de saúde brasileiro; um estudo foi realizado com os profissionais de saúde e não com os usuários do serviço público de saúde brasileiro; 11 estudos não abordavam o sistema de saúde público de saúde brasileiro como cenário; um estudo trabalhava a questão cultural da população considerada terceiro gênero; um estudo discutia o modo de transmissão de IST/AIDS; e dois por serem discussões teóricas sobre 0 assunto). Por fim, após todos os processos de refinamento das fontes localizadas na busca quatro artigos foram recuperados para análise - por responderam à pergunta norteadora do trabalho. Destes quatro artigos três foram recuperados da base de dados LILACS com os conjuntos de unitermos 2.1 e 5 e um artigo foi recuperado da base de dados PePSIC com o conjunto de unitermos 2.1. Todo o processo, tanto de busca, seleção quanto de recuperação das fontes encontra-se detalhado na Figura 1.

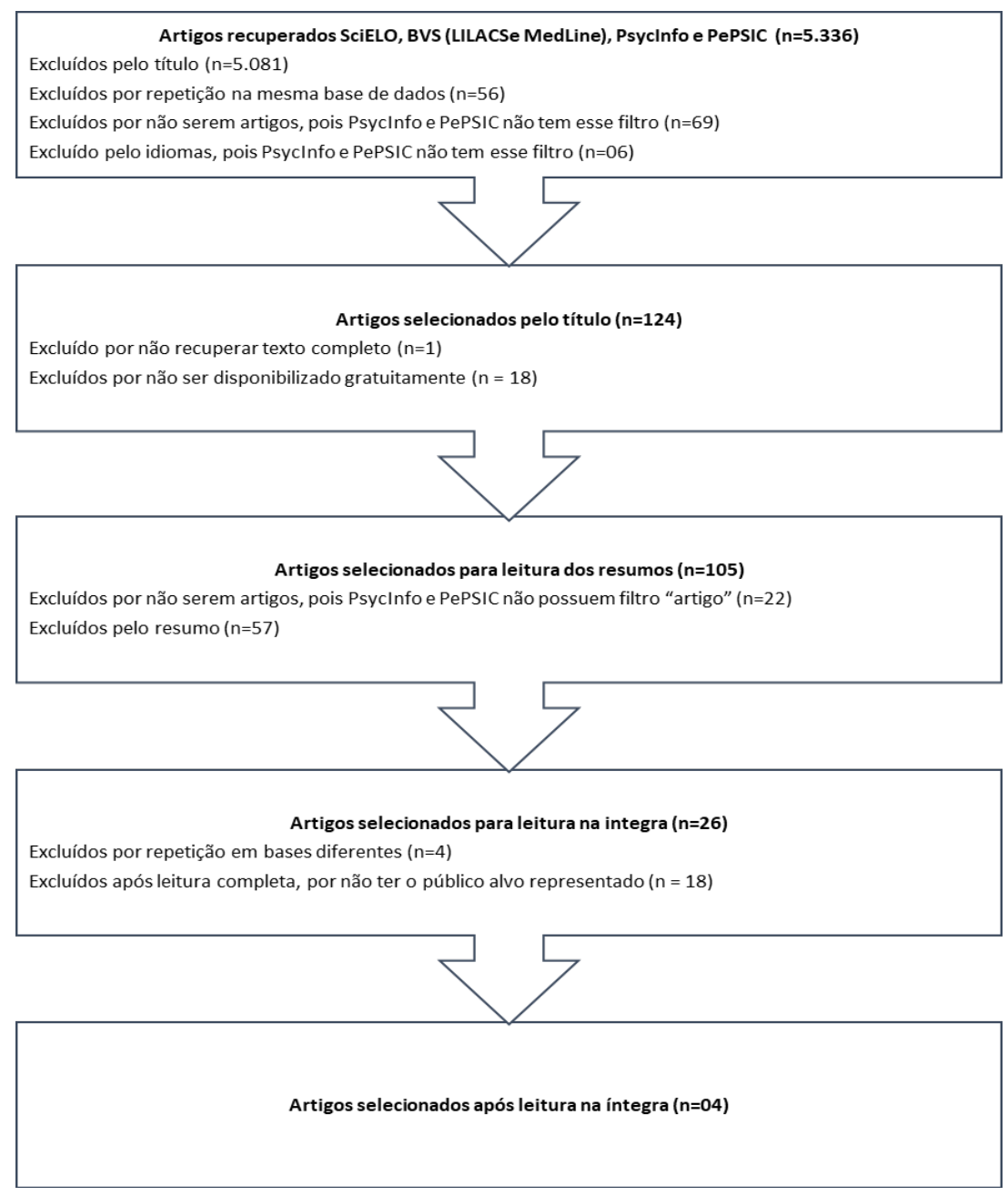

FIGURA 1.

Fluxograma representando os procedimentos do processo de composição do corpus. 
Caracterização dos artigos recuperados

A amostra final desta revisão constitui-se por quatro artigos científicos selecionados pelos critérios de inclusão/exclusão previamente estabelecidos e que incluíram a população transgênera dentro de suas pesquisas e respondiam à questão norteadora desse trabalho. As temáticas e estratégias utilizadas, de um modo breve, foram: população LGBT incluindo travestis e mulheres transexuais separadamente (primeiro artigo/A1); população LGBT, mas somente travestis compondo a amostra (segundo artigo/A2); amostra composta por transexuais e travestis (terceiro artigo/A3); 0 quarto e último artigo (A4) continha exclusivamente travestis na sua amostra. As principais análises dos artigos serão apresentadas a seguir, em seções específicas.

Resgatado na base de dados LILACS através do conjunto de unitermos 5 ([transexuais OR transsexuals] AND sistema único de saúde $O R$ unified health system) 0 artigo A1 (Ferreira, Pedrosa, \& Nascimento, 2018) foi publicado no periódico Revista Brasileira em Promoção da Saúde, cujo objetivo foi apreender as dimensões do acesso e da Atenção Integral na rede do SUS na perspectiva da diversidade de gênero, utilizando a abordagem qualitativa como método de pesquisa e realizada em Unidades Básicas de Saúde (USB) a partir de grupos focais, dos quais participaram 19 pessoas da comunidade LGBT durante 0 ano de 2016 na cidade de Teresina/Piauí. 0 artigo apresenta as entrevistas de 11 transgêneros (seis travestis e cinco mulheres transexuais) sem divulgação das idades dos participantes.

0 artigo A2 (Garcia, Albuquerque, \& Adami, 2016) resgatado da PePSIC com os conjuntos de unitermos 2.1 (pessoas transgênero OR transgender persons AND saúde $\mathrm{OR}$ health) foi publicado no periódico Journal of Human Growth and Development, cujo objetivo foi 0 de identificar as representações sociais e principais desigualdades, obstáculos e desafios vivenciados pela população de lésbicas, gays, bissexuais, travestis e transexuais com relação ao acesso aos serviços de saúde na cidade de Juazeiro do Norte/Ceará no período de julho a setembro de 2013 e utilizou a abordagem qualitativa de entrevista a partir da técnica de snowball sample (cada participante da pesquisa indica contatos de outros possíveis sujeitos). 0 estudo foi realizado em parceria com uma instituição que milita politicamente na cidade em prol dos direitos humanos do grupo LGBT. Dos 30 entrevistados somente 9 eram travestis com idade entre 18 a 51 anos.

Resgatada da base de dados LLILACS através do conjunto de unitermos 2.1 (pessoas transgênero OR transgender persons AND saúde OR health) a pesquisa do artigo A3 (Rocon, Rodrigues, Zamboni, \& Pedrini, 2016) foi publicada no periódico Ciência \& Saúde Coletiva, cujo objetivo foi identificar representações sociais de saúde e principais desigualdades, obstáculos e desafios pela população LGBT com relação ao acesso em saúde (na região metropolitana da grande Vitória/ES), utilizou a abordagem qualitativa por meio de entrevistas semiestruturadas com 15 pessoas transgêneras (travestis, mulheres e homens transexuais). A investigação foi realizada na sede de associação capixaba de redução de danos (ACARD) no Campus da Universidade Federal do Espírito Santo (UFES), no ambulatório de Urologia do Hospital Universitário (HUCAM) e na residência de alguns entrevistados. Porém, 0 ano da realização da coleta de dados da pesquisa não foi informado no artigo.

O último artigo A4 (Souza \& Pereira, 2015) foi resgatado da base de dados LILACS através dos unitermos 2.1 (pessoas transgênero OR transgender persons AND saúde $O R$ health) teve como objetivo apresentar os cuidados com a saúde de travestis do município de Santa Maria/RS e foi publicado no periódico Texto \& Contexto Enfermagem. A pesquisa foi realizada entre janeiro e novembro de 2012 com 49 travestis 
em busca de cuidados com a saúde na cidade de Santa Maria/RS através do método qualitativo de pesquisa por meio de investigação etnográfica (entrevistas, observação participante e acompanhamento da vida cotidiana). As idades das participantes variavam entre 18 e 53 anos. Todas essas informações estão dispostas na Tabela 1 e 2.

TABELA 1.

Descrição e identificação do conteúdo amostral selecionado para a revisão.

\begin{tabular}{|c|c|c|c|c|c|c|}
\hline ID & Lugar & Amostra & $\begin{array}{l}\text { Transexuais } \\
\text { Participantes }\end{array}$ & $\begin{array}{l}\text { Coleta de dados } \\
\text { (local e estratégia) }\end{array}$ & $\begin{array}{c}\text { Idade } \\
\text { participantes }\end{array}$ & $\begin{array}{c}\text { Período da } \\
\text { pesquisa }\end{array}$ \\
\hline A1 & Piauí, Brasil & 19 & 11 & Unidade Básica de saúde/ Grupos Focais & $\begin{array}{c}\text { Não } \\
\text { informado }\end{array}$ & Ano de 2016 \\
\hline A2 & Ceará, Brasil & 30 & 09 & Não informado/ Entrevista & 18 a 51 & $\begin{array}{c}\text { Julho a } \\
\text { setembro/ } 2013\end{array}$ \\
\hline A3 & $\begin{array}{c}\text { Espírito } \\
\text { Santo, Brasil }\end{array}$ & 15 & 15 & $\begin{array}{l}\text { Sede ACARD, Campus UFES, Ambulatório } \\
\text { do HU e na residêncial } \\
\text { Entrevista e questionário socioeconômico }\end{array}$ & 24 a 53 & Não informado \\
\hline A4 & $\begin{array}{l}\text { Rio Grande } \\
\text { do Sul, Brasil }\end{array}$ & 49 & 49 & $\begin{array}{l}\text { Pensionato, pontos de prostituição, casas } \\
\text { de Santo, desfiles de carnaval, paradas } \\
\text { LGBT/ Observação participante, entrevista } \\
\text { em profundidade e acompanhamento da } \\
\text { vida cotidiana }\end{array}$ & 18 a 53 & $\begin{array}{c}\text { Janeiro a } \\
\text { novembro/ } 2012\end{array}$ \\
\hline
\end{tabular}

TABELA 2.

Principais resultados dos artigos recuperados.

\begin{tabular}{|c|c|c|}
\hline ID & Objetivos & Principais resultados \\
\hline A1 & $\begin{array}{l}\text { Apreender as dimensões do acesso e da atenção } \\
\text { integral na rede do Sistema Único de Saúde (SUS) na } \\
\text { perspectiva da diversidade de gênero. }\end{array}$ & $\begin{array}{l}\text { Emergência no acesso às redes de atenção à saúde } \\
\text { integral da população LGBT com o intuito de promover } \\
\text { a inclusão em seus diversos equipamentos sociais. } \\
\text { Facilitar práticas de equidade. }\end{array}$ \\
\hline $\mathrm{A} 2$ & $\begin{array}{l}\text { Identificar Representações Sociais de saúde e } \\
\text { principais desigualdades, obstáculos e desafios } \\
\text { vivenciados pela população de Lésbicas, Gays, } \\
\text { Bissexuais, Travestis e Transexuais com relação ao } \\
\text { acesso aos serviços de saúde. }\end{array}$ & Obstáculos no acesso aos serviços de saúde. \\
\hline A3 & $\begin{array}{l}\text { Discutir as dificuldades de pessoas trans moradoras } \\
\text { da região metropolitana da Grande Vitória/ES em } \\
\text { acessarem os serviços de saúde no SUS. }\end{array}$ & $\begin{array}{l}\text { Importância de elaborar programas de educação e } \\
\text { campanhas permanentes sobre o direito de acesso ao } \\
\text { sistema de saúde livre de discriminação e com uso do } \\
\text { nome social. }\end{array}$ \\
\hline A4 & $\begin{array}{l}\text { Apresentar os cuidados com a saúde de travestis do } \\
\text { município de Santa Maria, Rio Grande do } \\
\text { Sul, Brasil. }\end{array}$ & $\begin{array}{l}\text { Pouca adesão aos serviços institucionalizados de } \\
\text { saúde, optando por outras formas de cuidado. }\end{array}$ \\
\hline
\end{tabular}

Os principais resultados (apresentados na Tabela 2) ressaltados em cada um dos quatro artigos destacaram: a necessidade de melhorar 0 acesso às redes de atenção à saúde integral da população LGBT com 0 intuito de promover a inclusão em seus diversos equipamentos sociais, promulgar 0 respeito e facilitar práticas de equidade; a existência de obstáculos no acesso aos serviços de saúde; a importância de elaborar 
programas de educação e campanhas permanentes sobre 0 direito de acesso ao sistema de saúde livre de discriminação e com uso do nome social; a evasão dos usuários dos serviços institucionalizados de saúde, optando por outras formas de cuidado.

\section{Descrição qualitativa}

Os artigos foram agrupados em três categorias temáticas a posteriori mediante a semelhança dos seus conteúdos, a saber: 1) atendimento no SUS, 2) uso do nome social, e 3) IST/ AIDS - conforme pode ser observado na Tabela 3.

TABELA 3.

Tabulação por artigo e categorias a serem analisadas.

\begin{tabular}{|c|c|}
\hline ID & Categorias \\
\hline $\mathrm{A} 1$ & $\begin{array}{c}\text { Atendimento no SUS } \\
\text { Uso do nome social } \\
\text { IST/AIDS }\end{array}$ \\
\hline A2 & $\begin{array}{c}\text { Atendimento no SUS } \\
\text { IST/AIDS }\end{array}$ \\
\hline A3 & $\begin{array}{c}\text { Atendimento no SUS } \\
\text { Uso do nome social } \\
\text { IST/AIDS }\end{array}$ \\
\hline A4 & $\begin{array}{c}\text { Atendimento no SUS } \\
\text { Uso do nome social } \\
\text { IST/AIDS }\end{array}$ \\
\hline
\end{tabular}

\section{Categoria 1 - Atendimento no SUS.}

Os quatro artigos abordaram a percepção da população transgênera em relação ao atendimento recebido no SUS (Ferreira, Pedrosa, \& Nascimento, 2018; Garcia, Albuquerque, \& Adami, 2016; Rocon, Rodrigues, Zamboni, \& Pedrini, 2016; Souza \& Pereira, 2015). Os relatos dos entrevistados naqueles artigos apontaram questões diversas, tais como o incômodo pela forma como são tratadas as pessoas transgêneras nos serviços de saúde (sobretudo pelo ajuizamento moral e social) e o distanciamento evidenciado por meio de gestos, olhares preconceituosos e falas dos profissionais de saúde do SUS.

Os participantes das pesquisas destacaram que (devido à atuação dos profissionais e seu despreparo), tentam evitar a discriminação abandonando 0 serviço de saúde e acabam se automedicando. No relato de uma travesti do artigo (A4) foi mencionado que esta acabou optando por tratamento particular quando precisa de algum cuidado médico, pois quando paga pelo tratamento acaba sendo efetivamente respeitada. No mesmo artigo, outra travesti relatou que às vezes prefere se tratar em casa a ir ao serviço público de saúde para evitar olhares, ironias e ser chamada pelo nome do registro do nascimento (que não condiz com seu nome social nem com sua autodeterminação de gênero).

Nos artigos foi destacado, ainda, que comumente ocorre discriminação por parte de trabalhadores da saúde (incluindo recepcionistas, vigilantes e integrantes das equipes médicas) em relação à população LGBT e que, segundo a perspectiva das participantes, está associado ao despreparo destes profissionais para lidar com as diferentes demandas desses sujeitos (Ferreira, Pereira, Tajra, Araújo, Freitas, \& Pedrosa, 2017). 
Massignam, Bastos e Nedel (2015) apontaram que muitas pesquisas evidenciam que as experiências discriminatórias nos contextos de saúde acarretam iniquidades e efeitos negativos para a saúde. Com a população LGBT não é diferente, tanto que em 2011 o Ministério da Saúde lançou a Portaria $n^{0} 2.836$ instituiu a Política Nacional de Saúde Integral de Lésbicas, Gays, Bissexuais, Travestis e Transexuais. Segundo Massignam et al. (2015) essas ações e políticas não asseguram a esses grupos o fim da discriminação nos serviços públicos de saúde, entretanto, ao criar barreiras e constrangimentos na execução da discriminação (que, não raro, ocasiona a exclusão de um direito universal) há maior visibilidade das necessidades das populações transgêneras. Deste modo, constitui uma modalidade de intervenção que incide diretamente sobre as normas culturais vigentes em uma determinada sociedade com iniciativas voltadas para a superação de práticas discriminatórias.

Contudo, Massignam et al. (2015) ressaltaram que é necessário lembrar que essas políticas só se viabilizam quando traduzidas à realidade específica local, exigindo um esforço conjunto, sob a perspectiva de cada um e de todos os gestores, técnicos, conselheiros e demais envolvidos na produção do cuidado em saúde - ou seja, trata-se de uma orientação político-institucional específica e deliberada na área da Saúde. Assim, tais ações só se efetivam com um trabalho conjunto para promover a saúde integral da população LGBT, eliminando a discriminação e 0 preconceito institucional e contribuindo para a redução das desigualdades e para a consolidação do SUS como sistema universal, integral e equitativo, como assegurado pela Política Nacional de Saúde Integral LGBT (Brasil, 2011).

Para Melo et al. (2011) os problemas enfrentados pela população LGBT, no que diz respeito ao acesso a serviços de saúde, são ainda mais específicos nos casos de travestis e transexuais. Por exemplo, por reivindicarem atendimentos especializados para demandas que não se colocam para outros segmentos populacionais - tais como as alterações corporais associadas ao uso de hormônios e silicone industrial por conta própria, ou seja, sem acompanhamento médico especializado, acarretando riscos graves à saúde como acidente vascular cerebral (AVC), aneurismas e ulcerações com carcinomas no caso do uso do silicone industrial (Calderaro et al., 2008). Entretanto, os profissionais de saúde, como relataram Calderaro et al. (2008), acabam por culpabilizar homens/mulheres transexuais e as travestis pelos adoecimentos e/ou problemas de saúde, como se estes fossem uma punição por transgredirem o binarismo heteronormativo que a sociedade tenta impor como normal.

No ano de 2008 o Ministério da Saúde publicou um texto de difusão técnicocientífica (Brasil, 2008), no qual foram discutidas ações voltadas para a promoção de ações diferenciadas em saúde, cujo objetivo era reduzir as desigualdades de grupos em condições de exclusão social e iniquidade em saúde. 0 combate à homofobia e à transfobia é uma das estratégias pretendidas para garantir 0 acesso aos serviços de saúde visando à qualidade da atenção prestada. Isso ocorre porque dentre os princípios do SUS estão a universalidade, a partir do qual todos têm acesso gratuito aos serviços de saúde, e a integralidade, segundo a qual devem ser ofertadas todas as modalidades de atendimentos disponíveis, compreendendo desde iniciativas e ações de promoção e prevenção até as intervenções especializadas em saúde (Brasil, 2008).

A capacitação dos profissionais de saúde acerca das práticas sexuais e sociais da população LGBT é uma das políticas de enfrentamento da iniquidade, que requer iniciativas constantes e estratégias inovadoras, segundo o Ministério da Saúde (Brasil, 2008), pois só assim a atenção e 0 
cuidado em Saúde serão condizentes com as legítimas necessidades e demandas dessa população.

\section{Categoria 2 - Uso do nome social.}

Publicados entre 2015 e 2018 três artigos (Ferreira, Pedrosa, \& Nascimento, 2018; Rocon, Rodrigues, Zamboni, \& Pedrini, 2016; Souza \& Pereira, 2015) abordaram o uso e respeito pelo nome social. Esta questão também se encontra presente nas discussões de Calderaro et al. (2008) ao discorrerem sobre uma mulher transexual que busca o pronto-socorro e já na recepção precisa mostrar 0 documento de identificação pessoal. Essa mulher passou pelo primeiro constrangimento por possuir uma aparência física e social feminina e ter 0 nome masculino e o sexo masculino indicados na carteira de identidade, sendo que 0 segundo desagrado ocorreu no momento da consulta por ter o nome de registro pronunciado em voz alta para todos os presentes, causando um desconforto e olhares a sua volta, olhares esse de estranheza e reprovação. Esse exemplo foi escrito em 2008, porém, uma década após, discute-se 0 mesmo modus operandis por parte de muitas equipes de saúde, mesmo sendo assegurado o preenchimento do nome social nos documentos de identificação do SUS (cartão do SUS e prontuário), como explicita a Carta dos Direitos dos Usuários do SUS (Brasil, 2006).

Os relatos dos artigos analisados (Ferreira et al., 2018; Rocon et al., 2016; Souza et al., 2015) apresentaram 0 desrespeito à utilização do nome social já no acolhimento dos serviços, a começar pela exigência por parte do usuário que se the seja utilizado o nome social - haja vista que isso não é cumprido (segundo relato dos próprios participantes) pelos profissionais de saúde nas pesquisas citadas. 0 desrespeito ao nome social faz com que essa população, mesmo quando procure pelo serviço de saúde, acabe não aguardando pelo ou aderindo ao atendimento pelo fato de ser chamado pelo nome do registro, o que the gera diversos constrangimentos. Esse desrespeito, em muitas situações, acaba inviabilizando que essa população busque pelo serviço de saúde.

$\mathrm{Na}$ área da Saúde, desde 2006, nos documentos e prontuários é destinado um campo específico para o preenchimento do nome social do usuário (Brasil, 2006). Entretanto, ainda falta em muitas situações e serviços, por parte dos profissionais da saúde, respeitarem o nome social tanto no acolhimento quanto nos atendimentos. Essa ação diminuiria, senão impediria, a ocorrência de constrangimentos que podem gerar uma barreira ao acesso a esses serviços e evasão do mesmo. Para Silva, Silva, Coelho e Martiniano (2017) a viabilização do uso do nome social, tanto oralmente como nos documentos e prontuários oficiais dos serviços de saúde, também viabilizaria caminhos para a concretização da atenção integral a saúde, pois 0 transgênero que estiver buscando 0 serviço de saúde irá se sentir acolhido e respeitado naquele espaço, possibilitando a adesão ao tratamento e o prosseguimento nas consultas e tratamentos necessários.

Segundo Silva et al. (2017), ao se sentir reconhecido, compreendido em sua autodeterminação de identidade de gênero e, principalmente, respeitado, o usuário sente-se confortável para acessar os serviços e usufruir de seus direitos em saúde, além de se sentir inserido na sociedade. Os processos de atenção e de cuidados em Saúde, a permanência no serviço e a aderência aos cuidados passa pelo uso do nome social, pois as travestis e as pessoas transexuais sentem-se acolhidas nas ações de promoção do acesso aos serviços de saúde a partir do estabelecimento de vínculos profissionalpaciente e instituição-paciente (Silva et al., 2017). Além de que estimularia o respeito à diversidade, aboliria a violação de direitos na esfera da Saúde e reafirmaria 0 compromisso de universalidade e equidade do SUS que passa pela ordem do respeito ao nome social. 
Categoria 3 - IST/AIDS.

A temática da população transgênera com as IST e a AIDS são abordadas nos quatro artigos (Ferreira, Pedrosa, \& Nascimento, 2018; Garcia, Albuquerque, \& Adami, 2016; Rocon, Rodrigues, Zamboni, \& Pedrini, 2016; Souza \& Pereira, 2015). Segundo Calderaro et al. (2008) em meados da década de 1990, - Governo Federal estabeleceu o Programa Nacional de Doenças Sexualmente Transmissíveis e AIDS (PNDST/AIDS), por intermédio do Ministério da Saúde, devido à epidemia do HIVIAIDS que atingiu o País. 0 Governo Federal Brasileiro começou a dirigir esforços de atenção à saúde às primeiras vítimas dessa epidemia, que incluía também as travestis e transexuais. Porém, Calderaro et al. (2008) apontaram que a saúde da população transgênera não pode ser restrita ao campo da IST/AIDS, pois, segundo os autores, todas as doenças físicas e psíquicas podem atingir a população como um todo, além de que ao buscarem cuidados de saúde se depararem com situações que não ocorreriam na vida de quem está inserido no binarismo heteronormativo. Segundo Butler (2016), pessoas transgêneras e transexuais não vivenciam as discriminações, constrangimentos, preconceitos institucionais e ajuizamentos morais como as pessoas cisgêneras, estando mais expostas às vulnerabilidades e riscos psicossociais.

Segundo Melo et al. (2011) as transexuais e travestis não buscam os serviços de saúde apenas quando inseridas no processo de readequação sexual ou em consequência da infecção do HIVIAIDS. Contudo, muitas vezes 0 acesso da população LGBT aos serviços de saúde tem como porta de entrada principal e quase única os ambulatórios especializados em HIVIAIDS, "confinando" as travestis e transexuais aos ambulatórios específicos e, em alguns casos, as aborda como infectantes (às vezes, sem realmente serem) já que os serviços estão interligados às demandas de IST e AIDS. Os mesmos autores comentam que no serviço de profilaxia pós-exposição não ocorre discriminação como exemplificado no A3. Porém, o artigo $A 4$, por sua vez, uma participante travesti relatou que 0 atendimento médico foi realizado com a porta aberta no serviço de testagem anti-HIV e ela se sentiu muito constrangida com este fato, além de também não respeitarem seu nome social, fazendo com que fosse embora sem realizar os exames necessários.

Para Melo et al. (2011) existe um desafio em assegurar que as práticas já desenvolvidas nas ações relativas ao tratamento de pessoas com IST/AIDS sejam expandidas para todos os âmbitos de ações do SUS de maneira a garantir que travestis e transexuais encontrem atendimentos de qualidade quando buscarem cuidados para sua saúde, independentemente de sua orientação sexual e identidade de gênero, e também independente de seu estado sorológico para o HIV.

É preciso reiterar que a porta de entrada para o sistema de saúde muitas vezes acaba sendo o ambulatório de IST/AIDS, pelo fato de a população transgênera se encontrar em situação de vulnerabilidade e risco social, uma vez que as instituições escolares e a própria família não raro os expulsam (desses locais, que deveriam ser espaços de proteção social e pessoal) devido à transfobia. Com pouca ou nenhuma escolaridade, sem um lar e com uma fragilizada rede de cuidados e proteções, resta a muitos transgêneros e travestis a prostituição como atividade geradora de renda, 0 que aumenta 0 risco de contaminações (e de transmissões) de IST/AIDS - estabelecendo um círculo danoso.

Como referido nas categorias precedentes ("Atendimento no SUS" e "Uso do nome social") a capacitação dos profissionais de saúde sobre as práticas sexuais, sociais e questões de gênero (Silva et al., 2017) da população LGBT é uma das políticas de enfrentamento da iniquidade que 
requer iniciativas constantes e estratégias inovadoras e assertivas. Segundo 0 Ministério da Saúde (Brasil, 2008) só assim o cuidado e a atenção à saúde serão condizentes com as reais necessidades $e$ demandas (em saúde) dessa população para ultrapassar a equivocada associação dos processos de saúde/doença dessa população à epidemia de IST/AIDS.

\section{Considerações Finais}

Algumas pesquisas vêm sendo desenvolvidas em torno da questão dos atendimentos em saúde sob a perspectiva das pessoas transgêneras, porém poucas a têm abordado a partir da perspectiva dos(as) próprios(as). Foi possível essa identificação quando se observa que dentre os (22) artigos que permaneceram para leitura na íntegra, a ampla maioria (18) foram excluídos por ou analisar 0 atendimento pela perspectiva dos profissionais de saúde e não dos usuários; ou discutir a questão da violência sem abordar a questão na área da Saúde; ou analisar a questão da transmissão do IST/AIDS nessa população especifica, mas não o atendimento em si; ou abordar a questão cultural do considerado terceiro gênero; ou discutir somente no plano teórico os atendimentos na área da saúde; ou, por ainda e por fim, não abordar o SUS como local ou cenário da pesquisa.

A respeito da presente revisão algumas limitações podem ser apontadas. Dentre elas o pouco número de artigos recuperados é 0 mais significativo, uma vez que o número de quatro artigos não permite generalizações; assim como a utilização de apenas artigos disponibilizados gratuitamente e a não inclusão de outras formas de fontes (livros, capítulos, dissertações, teses, editoriais, comunicações em eventos científicos dentre os quais certamente se espera que alguns serão comunicados em formato de artigos científicos nos próximos anos). Por outro lado, essa mesma limitação aponta e reforça a premente necessidade em explorar esse campo de investigação, pois evidencia a relevância de ampliar e aprofundar a investigação na área do atendimento em Saúde tendo como público-alvo a população transgênera.

Numa apreciação ampla os artigos analisados evidenciam a importância da formação continuada dos profissionais da Saúde, bem como dos investimentos públicos para o desenvolvimento dessas capacitações e conscientização dos profissionais sobre as questões de gênero, ao enfatizar 0 respeito pela utilização do nome social como ferramenta política para a inclusão de travestis e transexuais no processo de atenção e de cuidado em Saúde no âmbito do sistema público de saúde. Além disso, também é necessário refletir sobre a necessidade de transformar as representações tradicionalistas e moralistas de gênero e sexualidade vigentes de maneira que os estereótipos e representações de gênero não causem maiores problemas e consequências para as pessoas que não se encaixam no binarismo homem/mulher e que seja permitido a cada ser humano se expressar no mundo de maneira que the parecer mais adequado. Trata-se, em última instância, de uma discussão não sobre moral sexual, mas sim da garantia de direitos fundamentais por parte de todo e qualquer cidadão.

Por fim, é indispensável compreender o que esses sujeitos pensam a respeito de processos que lhes dizem respeito, de modo a incluí-los na discussão e tomadas de decisões pertinentes a esse tema, o que estimula a realização de novos estudos e investigações acerca das percepções dos transgêneros sobre 0 atendimento nos serviços de saúde público com o intuito de identificar suas demandas, necessidades, especificidades, constrangimentos e dificuldades. Apenas isso pode melhorar, qualificar, aprimorar e facilitar o acesso à atenção e aos cuidados em saúde dessa população em especial, e também de todos os cidadãos ao sistema público de saúde. 
REFEREANCIAS

Arán, M. \& Peixoto Júnior, C. A. (2007). Subversões do desejo: sobre gênero e subjetividade em Judith Butler. Cadernos Pagu, 28, 129-147. DOI: 10.1590/S0104-83332007000100007

Bento. B. (2017). O que é transexualidade. Taubaté, São Paulo: Brasiliense.

Brasil. (1988). Constituição da República Federativa do Brasil de 1988. Recuperado de http://www.planalto.gov.br/ccivil03/cons tituicao/constituicaocompilado.htm

Brasil. Ministério da Saúde. (2016). Decreto Presidencial $n^{0} 8901$ de 10 de novembro de 2016. Publicado no DOU de 11 de novembro de 2016. Recuperado de https://www.planalto.gov.br/ccivil_03/_a to2015-2018/2016/decreto/d8901.htm

Brasil. Ministério da Saúde. (2006). Portaria n675 de 30 de março de 2006. Carta dos direitos dos usuários da saúde. Recuperado de http://www.saude.pr.gov.br/arquivos/Fil e/CIB/LEGIS/PortGMMS_675_30marco _2006_carta_dos_direitos.pdf

Brasil. Ministério da Saúde. (2008). Saúde da população de gays, lésbicas, bissexuais, travestis e transexuais. Revista de Saúde Pública, 42(3), 570573.

Brasil. Ministério da Saúde. (2011). Portaria $n^{0} 2.836$, de $1^{\circ}$ de dezembro de 2011. Política Nacional de Saúde Integral de Lésbicas, Gays, Bissexuais, Travestis e Transexuais. Recuperado de http://bvsms.saude.gov.br/bvs/saudeleg is/gm/2011/prt2836_01_12_2011.html

Butler, J. (2014). Regulações de Gênero. Cadernos Pagu, 42, 249-274. DOI: 10.1590/0104-8333201400420249

Butler, J. (2016). Problemas de Gênero: Feminismo e subversão da identidade. Rio de Janeiro: Civilização Brasileira.

Calderaro, F., Fernandes, B., \& Mello, L. (2008). Cidadania TTLBG e o direito à saúde no Brasil. In: D. G. Rocha, J. R. Lima, L. Mello, \& I. V. Batista (Org.), Diversidade e equidade no SUS: parceria universidade e educação popular. (p. 57-68). Goiânia, GO: Cânone Editorial.

Ercole, F. F., Melo, L. S., \& Alcoforado, C. L. G. C. (2014). Revisão integrativa versus Revisão sistemática. Revista Mineira de Enfermagem, 18(1), 9-11. DOI: 10.5935/1415-2762.20140001

Ferreira, B. O., Pedrosa, J. I. S., \& Nascimento, E. F. (2018). Diversidade de gênero e acesso ao sistema único de saúde. Revista Brasileira em Promoção da Saúde, 31(1), 1-10. DOI: 10.5020/18061230.2018.6726

Ferreira, B. O., Pereira, E. O., Tajra, F. O., Araújo, Z. A. M., Freitas, F. R. N. N., \& Pedrosa, J. I. S. (2017). Caminhos e vivências de investigação acerca da saúde da população LGBT em uma capital do nordeste brasileiro. Tempus, Actas de Saúde Coletiva, 11(1), 41-49. DOI: 10.18569/tempus.v1111.1855

Garcia, C. L., Albuquerque, G. A., \& Adami, F. (2016). Saúde de minorias sexuais do nordeste brasileiro: Representações, comportamentos e obstáculos. Journal of Human Growth and Development, 26(1), 94-100. DOI: 10.7322/jhgd.110985

Lionço, T. (2009). Atenção integral à Saúde e diversidade sexual no Processo Transexualizador do SUS: avanços, impasses, desafios. Psysis: Revista de Saúde Coletiva, 19(1), 43-53. DOI: 10.1590/S0103-73312009000100004

Massignam, F. M., Bastos, J. L. D. \& Nedel, F. B. (2015). Discriminação e saúde: um problema de acesso. Revista Epidemiologia e Serviços de Saúde, 24(3), 541-544. DOI: 10.5123/S167949742015000300020

Melo, L., Perilo, M., Braz, C. A., \& Pedrosa, C. (2011). Políticas de saúde para lésbicas, gays, bissexuais, travestis e transexuais no Brasil: em busca de universalidade, integralidade e equidade. Sexualidad, Salud $y$ Sociedad, 9, 7-28. DOI: 10.1590/S198464872011000400002

Mendes, K. D. S., Silveira, R. C. C. P., \& Galvão, C. M. (2008). Revisão 
integrativa: métodos de pesquisa para a incorporação de evidências na saúde e na enfermagem. Texto \& Contexto Enfermagem, 17(4), 758-764. DOI: 10.1590/S0104-07072008000400018

Rocon, P. C., Rodrigues, A., Zamboni, J., \& Pedrini, M. D. (2016). Dificuldades vividas por pessoas trans ao sistema único de saúde. Ciência \& Saúde Coletiva, 21(8), 2517-2525. DOI: 10.1590/1413-81232015218.14362015

Santos, C. M. C., Pimenta, C. A. M., \& Nobre, M. R. C. (2007). A estratégia PICO para a construção da pergunta de pesquisa e busca de evidências. Revista LatinoAmericana de Enfermagem, 15(3), 508511. DOI: 10.1590/S010411692007000300023

Silva, L. K. M., Silva, A. L. M. A., Coelho, A.A., \& Martiniano, C. S. (2017). Uso do nome social no sistema único de saúde: elementos para 0 debate sobre a assistência prestada a travestis e transexuais. Physis: Revista de Saúde
Coletiva, 27(3), 835-846. DOI: 10.1590/S0103-73312017000300023

Souza, M. H. T., \& Pereira, P. P. G. (2015). Cuidado com saúde: as travestis de Santa Maria, Rio Grande do Sul. Texto \& Contexto Enfermagem, 24(1), 146153. DOI: 10.1590/010407072015001920013

Souza, M. T., Silva, M. D., \& Carvalho, R. (2010). Revisão integrativa: o que é e como fazer. Einstein, 8(1), 102-106. DOI: 10.1590/s1679-45082010rw1134

Todos los trabajos publicados en

Revista Salud \& Sociedad (ISSNe:0718-7475) están sujetos a una licencia Creative Commons Reconocimiento 4.0 Internacional

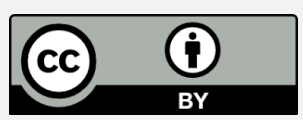




\section{RESUMO}

Objetivo: 0 presente estudo tem como objetivo apresentar uma revisão integrativa da literatura científica nacional e internacional (publicada no Brasil) sobre como os transgêneros percebem o atendimento realizado no serviço público de saúde. Método: A partir de uma questão norteadora elaborada segundo a estratégia PICO (acrônimo para Paciente, Intervenção, Comparação, Outcome/Desfecho) para realização de revisões sistemáticas da literatura científica, as bases de dados utilizadas foram SciELO, Lilas, MedLine, PePSIC e APA PsycNET ao combinar unitermos que estabelecessem relação com a temática. Resultado: Foram recuperadas 5336 fontes, sendo este total refinado seguindo os critérios de inclusão e exclusão (de título e de resumos) determinados pelos autores: incluir a população trans na sua pesquisa e responder à pergunta norteadora da pesquisa. Assim, quatro artigos foram totalmente recuperados para analisados de acordo com a semelhança de seu conteúdo em três categorias temáticas (atendimento no SUS, uso do nome social, infecções sexuais e AIDS), cujos principais resultados destacam as dificuldades desta população quanto à consolidação dos seus direitos na atenção/promoção à saúde pelo poder público. Conclusao: Ressalta-se a importância de se realizar mais estudos sobre a percepção das pessoas trans sobre os cuidados nos serviços públicos de saúde.

PALAVRAS-CHAVE: Pessoas transgênero, transexuais, saúde, sistema único de saúde.

\section{RESUMEN}

Objetivo: El objetivo de este estudio fue realizar una revisión sistemática de la literatura científica brasileña e internacional (publicada en Brasil) en el período comprendido entre 2008 y 2018 sobre cómo perciben los transexuales la atención prestada en los servicios de salud pública brasileña. Método: A partir de una pregunta orientadora establecida de acuerdo a la estrategia PICO (acrónimo de Paciente, Intervención, Comparación, Outcome/resultado) para revisiones de literatura sistemáticas las bases de datos SciELO, Lilas, MedLine, PePSIC y APA PsycNET fueron utilizadas combinando términos específicos. Resultados: Recuperamos 5,336 artículos de acuerdo con criterios de inclusión y exclusión entre los cuales debían incluir a la población transgénero en su investigación y responder a la pregunta guía de la investigación. Analizamos cuatro artículos en tres categorías temáticas: atención del SUS (sistema público de salud brasileño), uso del nombre social, infecciones sexuales y SIDA. Conclusión: Los resultados resaltan las dificultades de esta población con respecto a la consolidación de sus derechos en la atención/promoción de la salud pública. Enfatizaos la importancia de más estudios sobre la percepción de las personas transgénero sobre la atención en los servicios de salud pública.

PALABRAS CLAVE: Personas transgénero, transexuales, salud, sistema único de salud. 